\title{
Performance Evaluation of Common Bean (Phaseolus vulgaris L.) Genotypes for Yield and Related Traits at Areka, Southern Ethiopia
}

\author{
Simon Yohannes, Gobeze Loha, and Mesfin Kebede Gessese \\ Department of Plant Sciences, College of Agriculture, Wolaita Sodo University, Wolaita Sodo, Ethiopia \\ Correspondence should be addressed to Mesfin K. Gessese; mesfin04@yahoo.com
}

Received 5 July 2019; Accepted 10 October 2019; Published 25 January 2020

Academic Editor: Gábor Kocsy

Copyright (c) 2020 Simon Yohannes et al. This is an open access article distributed under the Creative Commons Attribution License, which permits unrestricted use, distribution, and reproduction in any medium, provided the original work is properly cited.

\begin{abstract}
Common bean is a source of dietary protein and the second most important legume crop in Africa next to faba bean. In Ethiopia common bean is the most important legume as the source of protein and export commodity. Hence, development of commercial varieties is one of the major tasks to meet increasing demand of the stake holders. To this effect, understanding the genetic variability, heritability and association between grain yield and other agronomic traits is necessary for effective plant breeding program. In this context, a field experiment was conducted during 2016/2017 cropping season at Areka Agricultural Research Center in southern Ethiopia with the objective of evaluating common bean genotypes for yield and related traits and also estimate the variability present among the genotypes. Treatments consisted of thirty three common bean genotypes were laid out in a randomized complete block design (RCBD) with three replications. Common bean genotypes exhibited considerable variations for agronomic traits and grain yield. Majority of the traits; plant height, number of nodes, internode length, leaf area, LAI, biological yield, pods per plant, HI and HSW had higher PCV. Genotypic coefficient of variance (GCV) varied from $1.88 \%$ to $37.72 \%$ with the highest GCV recorded for HSW. Heritability in broad sense $\left(\mathrm{H}^{2}\right)$ ranged from $0.52 \%$ to $95.33 \%$ with the highest value observed for HSW. The present study revealed significant variation among genotypes for traits considered except few insignificant traits. In addition, almost all the genotypes were well adapted to the study area and hence, the high yielding genotypes could be directly used as seed sources for production of common bean and some of the genotypes with best diseases resistance reaction, and with high heritability can possibly be used in common bean improvement program.
\end{abstract}

\section{Introduction}

Common bean is a source of dietary protein and the second most important legume crop in Africa next to faba bean [1]. It is the third most important source of calories for lower income African households after cassava and maize $[1,2]$. In Ethiopia common bean is the most important legume as the source of protein and export commodity [3]. Common bean contains considerable amount of protein being high in lysine and a good source of energy making it a good complement staple in the diet $[4,5]$. It is predominantly cultivated for cash in the central rift valley, but in other parts it is a major staple food supplementing the protein source for the poor farmers who cannot afford to buy expensive meat [6].
Since common bean is cultivated in most parts of Ethiopia with a wide range of variation in altitude, rainfall, temperature, cropping system and socio-economic factors, it is essential to assess the pattern of character variations among and between accessions to resolve the problems in different regions and adaptation zones. Assessing diversity in these germplasm introductions can possibly enable to identify elite genotypes with the greatest novelty and thus are most suitable for rescue or incorporation into crop improvement programs. In plant breeding, diversity can be assessed in different ways [7]. Plant breeding is essentially selection among the variables. Thus, an insight into the magnitude of variability present in a crop species is important as it allows effective selection [8]. Indeed, in selection for yield, more emphasis has to be given on the 
attributes with low environmental variability. Classical methods of estimating diversity among groups of plants have relied chiefly upon morphological characters, which still play a central role in the analysis of genetic variability in crop species and their relatives [9]. Thus, this study was initiated with the objectives of estimating the genetic variability of Ethiopian common bean commercial cultivars and promising genotypes as well as to evaluate their performance for economically important quantitative and qualitative traits.

\section{Materials and Methods}

A field experiment was conducted during 2016/2017 cropping season at Areka Agricultural Research Center in southern Ethiopia. An approximate geographical coordinates of the site is $7^{\circ} 41^{\prime} \mathrm{N}$ latitude and $37^{\circ} 41^{\prime} \mathrm{E}$ longitude with an altitude of 1790 meters above sea level. Soil type of experimental site is classified as pyroclastic origion [10]. The mean annual rainfall is $1460 \mathrm{~mm}$ with a bimodal pattern which extends from March to September with the peak months from April to September. The mean minimum and maximum temperatures of the area are 15 and $26^{\circ} \mathrm{C}$, respectively.

2.1. Treatments and Experimental Design. Treatments consisted in twenty two varieties and eleven advanced lines of common bean making a total of 33 genotypes (SEC 20, SMC 22, SMC 21, SEC 23, ALB 204, Waji, Brazil 1, SAB 735, Awash Melka, Awash 2, Melkadima, SER 125, Red Wolaita, Remeda, Nasir, SER 119, Duristu, Dimtu, Awassa Dume, Ibado, Goberasha, Roba, Brazil 2, Awash1, Deme, SAB 632, Tatu, ALB 179, ALB 209, ALB 61, ALB 25, NVA 682, NVA317) were used in the trial. The treatments were laid out in a randomized complete block design (RCBD) with three replications. The blocks were folded in order to reduce field variability. Each plot was $2.4 \mathrm{~m}$ wide and $2 \mathrm{~m}$ long with growth area of $4.8 \mathrm{~m}^{2}$. Seeds were hand planted by placing two seeds per hill and thinned after emergence in order to maintain the proposed plant density in each plot. The inter- and intra-row spacing was 40 and $10 \mathrm{~cm}$, respectively. Experimental field was ploughed, pulverized and leveled in order to get smooth seedbed. The recommended NPS fertilizer was applied at planting time at a rate of $117 \mathrm{~kg} / \mathrm{ha}$. Urea was used as $N$ source and applied at rate of $41 \mathrm{~kg} / \mathrm{ha}$ at planting taking into consideration the $N$ content in NPS fertilizer. All crop management practices such as cultivation, weeding etc., carried out as desired during crop growing period.

2.2. Data Collection and Measurements. Plant parameters recorded were days to flowering, days to maturity, plant height, pod length, stem diameter, internod length, leaf area index (LAI), number of pods per plant, seeds per pod, grain yield, biomass yield, thousand seed weight (TSW) and harvest index (HI). Days to flowering was recorded as the number of days from planting to $50 \%$ of the plants exhibit flowering per plot. Days to physiological maturity was recorded when $50 \%$ of plants in the plot lose green color of pod. Plant height, stem diameter, internode length, number of pods per plant and seeds per pod were taken from five randomly selected plants per plot. Leaf area per plant was measured by taking five plants from middle rows after initiation of flowering by using ruler/meter and calculated by using methods adopted in Areka research center and the resulting value was multiplied by correction factor 0.733 [11]. Leaf area index (LAI) was calculated as the ratio of total leaf area per ten plants $\left(\mathrm{cm}^{2}\right)$ per area of land occupied by the plants. Grain yield was harvested from central rows by avoiding border effects and converted to $\mathrm{kg} / \mathrm{ha}$ after adjusting moisture content at $10 \%$. Biomass was determined as the sum of straw weighed and total grain yield. Harvest index (HI) is the ratio of grain to the total biomass and estimated as:

$$
\mathrm{HI}=\frac{\text { grain yield }}{\text { biological yield }} \text {. }
$$

The estimation of genetic parameters were done to identify and ascertain the genetic variability among the genotypes and to determine the extents of environmental effect on various characters. Variance components due to phenotype $\left(\sigma_{\mathrm{p}}^{2}\right)$, genotype $\left(\sigma_{\mathrm{g}}^{2}\right)$, the environment $\left(\sigma_{\mathrm{e}}^{2}\right)$ were calculated by adopting the following formula suggested by Burton and DeVence [12]:

$$
\begin{gathered}
\operatorname{Genotypic} \text { variance }\left(\boldsymbol{\delta}^{2} \mathrm{~g}\right)=\frac{\mathrm{MSg}-\mathrm{Mse}}{r}, \\
\text { Phenotypic variance }\left(\boldsymbol{\delta}^{2} \mathrm{p}\right)=\boldsymbol{\delta}^{2} \mathrm{~g}+\boldsymbol{\delta}^{2} \mathrm{e}
\end{gathered}
$$

Environmental Variance $\left(\boldsymbol{\delta}^{2} \mathrm{e}\right)=$ error mean square,

where, $\mathrm{MSg}=$ mean square due to genotypes, $\mathrm{MSe}=$ mean square due to error, $r=$ number of replication.

Phenotypic and genotypic coefficients of variances were calculated according to Singh [13] as:

$$
\mathrm{PCV}=\frac{\sqrt{\text { phenotypic variance }}}{\text { population mean for trait }} \text { or PCV }=\frac{\sigma_{\mathrm{p}}^{2}}{X} \times 100 \text {, }
$$

where $\mathrm{PCV}=$ phenotypic coefficient of variation

$$
\mathrm{GCV}=\frac{\sqrt{\text { genotypic variance }}}{\text { population mean for trait }} \text { or } \mathrm{GCV}=\frac{\sigma_{\mathrm{g}}^{2}}{X} \times 100 \text {, }
$$

where $\mathrm{GCV}=$ genotypic coefficients of variation, $X=$ the grand mean of a character.

Broad sense heritability $\left(H^{2}\right)$ was calculated for each trait by using the formula [14]:

$$
H^{2}(\%)=\frac{\sigma_{\mathrm{g}}^{2}}{\sigma_{\mathrm{p}}^{2}} \times 100,
$$

where, $H=$ heritability in broad sense, $\sigma_{\mathrm{g}}^{2}=$ genotypic variance, $\sigma_{\mathrm{p}}^{2}=$ phenotypic variance.

Genetic advance (GA) under selection, assuming the selection intensity of $5 \%$ was calculated as proposed by Johnson et al. [15] as follows:

$$
\mathrm{GA}=K \cdot \sqrt{\sigma_{\mathrm{p}}^{2}} \cdot \frac{\sigma_{\mathrm{g}}^{2}}{\sigma_{\mathrm{p}}^{2}}=K \cdot H * \sqrt{\sigma_{\mathrm{p}}^{2}},
$$


where: $\mathrm{GA}=$ expected genetic advance, $K=$ the selection differential ( $K=2.056$ at $5 \%$ selection intensity).

Genetic advance as percent of mean was calculated to compare the extent of predicted advances of different traits under selection, using the formula given by Falconer and Mackey [16]:

$$
\mathrm{GAM}=\frac{\mathrm{GA}}{X} \times 100,
$$

where, $\mathrm{GAM}=$ genetic advance as percent of mean, $\mathrm{GA}=$ genetic advance under selection, $X=$ mean value of a trait.

Both genotypic and phenotypic correlation, which the inherent association between two variables were estimated by the formula suggested by Weber and Moorthy [17].

$$
\operatorname{rg}(x y)=\frac{\mathrm{G} \operatorname{cov}(x \cdot y)}{\sqrt{\sigma_{\mathrm{g}}^{2} x \cdot \sigma_{\mathrm{g}}^{2} y}},
$$

where: $\mathrm{rg}=$ genotype correlation coefficient, $\mathrm{G} \operatorname{cov}(x \cdot y)=$ genotype co-variance between, $\sigma_{\mathrm{g}}^{2} x=$ genotype variance for variable $x, \sigma_{\mathrm{g}}^{2} y=$ genotype variance for variable $y$.

$$
\operatorname{rp}(x y)=\frac{\operatorname{Pcov}(x \cdot y)}{\sqrt{\sigma_{\mathrm{p}}^{2} x \cdot \sigma_{\mathrm{p}}^{2} y}},
$$

where: $\mathrm{rp}=$ phenotype correlation coefficient, $\operatorname{Pcov}(x . y)=$ phenotype co-variance between variable $x$ and $y, \sigma_{\mathrm{p}}^{2} x=$ phenotype variance for variable $x, \sigma_{\mathrm{p}}^{2} y=$ phenotype variance for variable $y$.

Data of individual mean of ten randomly selected plants from each replication were subjected to statistical analysis. All Quantitative data were subjected to analysis of variance using SAS statistical analysis software version 8 [18]. Significant treatment means were separated using the least significant difference (LSD) test at 5\% probability level.

\section{Results and Discussion}

3.1. Phenological Traits. Analysis of variance showed that genotypes were significantly differed for days to flowering and maturity Table 1. Generally days to flowering ranged from 43.0 to 56.3 and maturity from 75.0 to 92.0. Genotype SAB 632 flowered (43.0) and matured (75.0) early as compared to the other cultivars. On the other hand, genotype SEC 20 took the longest days to flowering (56.4) and maturity (92.2). Genotypes SEC 20, SMC 21, ALB 204, Awash 2, SER 125, Red Wolaita, SER 119, Dursitu, Dimtu, Awasa Dume, Ibado, Gobe Rashia, Roba, Awash 1, Deme, ALB 179, ALB 209, ALB 61, ALB 25 and NVA 517 took relatively longer days to flowering and physiological maturity. Conversely, genotypes SMC 22, Waji, Brazil 1, SAB 735, Awash Melka, Melka Dima, Ramada, Nasir, Awassa Dume, Brazil 2, SAB 632, Tatu and NVA 682 exhibited relatively shorter days to flowering and physiological maturity. The difference of 13.4 and 17.2 days was observed between the longest and shortest days to flowering and maturity, respectively. This is an indication that there was a wide range of variability among genotypes for days to flowering and maturity. Similar findings were reported by Kassaye [7], Shahid and Kamaluddin [19] and Fahad et al. [20] that significant difference was observed for days to flowering and physiological maturity in common bean genotypes.

3.2. Growth Traits. Analysis of variance indicated that genotypes were significantly differed for growth traits Table 1 . Plant height for genotypes ranged from the shortest $(25.7 \mathrm{~cm})$ for NVA 682 and the tallest plant $(56.3 \mathrm{~cm})$ for Nasir Table 1 . Similarly, stem diameter ranged from $3.00 \mathrm{~cm}$ to $5.7 \mathrm{~cm}$ with greatest for genotype SEC 20 and the least for Tatu. Number of nodes per stems is one of the growth traits varied from 5.33 to $9.00 \mathrm{~cm}$ with the greatest number of nodes per stem exhibited for ALB 61 and the lowest for Melka Dima. Internode length ranged from $4.00 \mathrm{~cm}$ which was the least for genotype ALB 209 to the greatest for genotype Nasir with mean internode length of $7.33 \mathrm{~cm}$. In line with this, leaf area and LAI were varied from $13.33 \mathrm{~cm}^{2}$ and 4.18 , respectively. Both parameters were greatest for genotype Deme and smallest for genotype Waji Table 1. This result showed that the growth traits measured were significantly differed due to the existence of inherent genetic variations among the genotypes.

3.3. Yield Components and Yield. Analysis of variance revealed that genotypes of common bean were significantly differed for yield components and yield Table 2 . The number of pods per plant and seeds per pod were varied from 5.33 to 22.67 and 3.00 to 5.72. The highest number of pods per plant (22.67) and seeds per pod were recorded for genotype SEC 20. The lowest number of pods per plant (5.33) and seeds per pod (3.00) were observed for genotype Tatu. Genotypes had variable HSW which ranged from 13.67 to $48.67 \mathrm{~g}$ where the highest HSW (48.67 g) was achieved from genotype Deme and the lowest HSW (13.67 g) was obtained from Awash Melka. Biological yield as affected by genotypes ranged from 4333 to $17670 \mathrm{~kg} /$ ha. The greatest biological yield $(17670 \mathrm{~kg} / \mathrm{ha})$ was recorded for genotype SEC 20) and the lowest ( $4333 \mathrm{~kg} / \mathrm{ha}$ ) was for Tatu. Indeed, the biological yield difference of $13337 \mathrm{~kg} / \mathrm{ha}$ achieved between the highest and the lowest genotypes. Grain yield for genotypes varied from 1147 to $4462 \mathrm{~kg} / \mathrm{ha}$. The highest grain yield $(4462 \mathrm{~kg} / \mathrm{ha})$ was obtained from genotype SEC 20 and the lowest $(1147 \mathrm{~kg} / \mathrm{ha})$ from genotype Awash Melka. Harvest index (HI), which is the physiological efficiency and ability of a crop for converting the total dry matter into economic yield [21]. Hence, HI reflects the proportion of assimilate distribution between economic and total biomass yield. It ranged from 0.10 to 0.17 with the highest $\mathrm{HI}(0.17)$ for genotype ALB 204 and the lowest HI (0.10) for Ramada.

\subsection{Variance Components}

3.4.1. Phenotypic and Genotypic Variations. Genotypic and phenotypic coefficients of variation are used to measure the variability that exists in a given population under consideration. Phenotypic variance varied from 0.05 for $\mathrm{HI}$ to 1282.70 for biological yield Table 3 . Higher phenotypic variance $(\geq 100 \%)$ was observed for plant height, biological yield and HSW. In line with this, higher magnitude of difference between genotypic and environmental variance was observed for the 
TABLE 1: Mean performance of genotypes of common bean for phenological and growth traits.

\begin{tabular}{|c|c|c|c|c|c|c|c|c|c|}
\hline SN & Genotypes & $\begin{array}{l}\text { Days to } \\
\text { flowering }\end{array}$ & $\begin{array}{l}\text { Days to } \\
\text { maturity }\end{array}$ & $\begin{array}{c}\text { Plant } \\
\text { height }(\mathrm{cm})\end{array}$ & $\begin{array}{l}\text { Stem diameter } \\
(\mathrm{mm})\end{array}$ & $\begin{array}{l}\text { Nodes per } \\
\text { stem }\end{array}$ & $\begin{array}{l}\text { Inter node } \\
\text { length }(\mathrm{cm})\end{array}$ & $\begin{array}{l}\text { Leaf area } \\
\left(\mathrm{cm}^{2}\right)\end{array}$ & LAI \\
\hline 1 & SEC 20 & $56.4^{\mathrm{a}}$ & $92.1^{\mathrm{a}}$ & $31.3^{b c}$ & $5.70^{\mathrm{a}}$ & $6.00^{\mathrm{b}-\mathrm{d}}$ & $5.33^{a-c}$ & $18.33^{\mathrm{c}-\mathrm{g}}$ & $3.39^{a-e}$ \\
\hline 2 & SMC 22 & $46.3^{\mathrm{e}-\mathrm{h}}$ & $75.0^{\mathrm{e}}$ & $29.0^{\mathrm{bc}}$ & $4.00^{\mathrm{d}-\mathrm{f}}$ & $5.67^{\mathrm{cd}}$ & $5.00^{\mathrm{bc}}$ & $15.33^{\mathrm{e}-\mathrm{g}}$ & $1.49^{g-j}$ \\
\hline 3 & SMC 21 & $53.0^{\mathrm{a}-\mathrm{e}}$ & $89.0^{\mathrm{a}-\mathrm{c}}$ & $34.0^{\mathrm{bc}}$ & $4.33^{\mathrm{c}-\mathrm{e}}$ & $6.67^{\mathrm{a}-\mathrm{d}}$ & $5.00^{\mathrm{bc}}$ & $21.33^{\mathrm{a}-\mathrm{g}}$ & $2.29^{b-j}$ \\
\hline 4 & SEC 23 & $53.0^{\mathrm{a}-\mathrm{e}}$ & $90.0^{\mathrm{a}-\mathrm{c}}$ & $29.7^{\mathrm{bc}}$ & $5.33^{\mathrm{ab}}$ & $5.67^{\mathrm{cd}}$ & $5.33^{\mathrm{a}-\mathrm{c}}$ & $15.00^{\mathrm{fg}}$ & $2.40^{b-j}$ \\
\hline 5 & ALB 204 & $52.3^{\mathrm{a}-\mathrm{e}}$ & $92.0^{\mathrm{a}}$ & $28.0^{\mathrm{C}}$ & $4.33^{\mathrm{c}-\mathrm{e}}$ & $6.33^{\mathrm{b}-\mathrm{d}}$ & $4.67^{\mathrm{c}}$ & $21.33^{\mathrm{a}-\mathrm{g}}$ & $2.93^{\mathrm{a}-\mathrm{g}}$ \\
\hline 6 & Waji & $45.3^{\mathrm{f}-\mathrm{h}}$ & $75.0^{\mathrm{e}}$ & $45.3^{\mathrm{a}-\mathrm{c}}$ & $4.33^{\mathrm{c}-\mathrm{e}}$ & $6.33^{\mathrm{b}-\mathrm{d}}$ & $7.00^{\mathrm{ab}}$ & $13.33^{\mathrm{g}}$ & $1.12^{\mathrm{j}}$ \\
\hline 7 & Brazil 1 & $43.7^{\mathrm{g}-\mathrm{h}}$ & $75.0^{\mathrm{e}}$ & $33.7^{\mathrm{bc}}$ & $3.67^{\mathrm{e}-\mathrm{g}}$ & $6.00^{\mathrm{b}-\mathrm{d}}$ & $5.33^{\mathrm{a}-\mathrm{c}}$ & $22.00^{\mathrm{a}-\mathrm{g}}$ & $1.57^{f-j}$ \\
\hline 8 & SAB -735 & $44.3^{\mathrm{g}-\mathrm{h}}$ & $75.0^{\mathrm{e}}$ & $34.3^{\mathrm{bc}}$ & $3.33^{\mathrm{fg}}$ & $6.00^{\mathrm{b}-\mathrm{d}}$ & $5.33^{a-c}$ & $20.7^{\mathrm{a}-\mathrm{g}}$ & $1.35^{\mathrm{h}-\mathrm{j}}$ \\
\hline 9 & $\begin{array}{l}\text { Awash } \\
\text { Melka }\end{array}$ & $44.0^{\mathrm{g}-\mathrm{h}}$ & $75.0^{\mathrm{e}}$ & $27.7^{\mathcal{C}}$ & $3.67^{\mathrm{e}-\mathrm{g}}$ & $5.67^{\mathrm{cd}}$ & $5.33^{\mathrm{a}-\mathrm{c}}$ & $13.34^{\mathrm{g}}$ & $1.22^{\mathrm{ij}}$ \\
\hline 10 & Awash 2 & $55.0^{\mathrm{a}-\mathrm{b}}$ & $91.3^{\mathrm{a}-\mathrm{b}}$ & $41.3^{\mathrm{a}-\mathrm{c}}$ & $4.33^{\mathrm{c}-\mathrm{e}}$ & $7.33^{\mathrm{a}-\mathrm{d}}$ & $6.00^{\mathrm{a}-\mathrm{c}}$ & $20.00^{\mathrm{b}-\mathrm{g}}$ & $2.05^{\mathrm{c}-\mathrm{j}}$ \\
\hline 11 & Melka Dima & $47.7^{\mathrm{c}-\mathrm{h}}$ & $87.7^{\mathrm{b}-\mathrm{c}}$ & $31.3^{\mathrm{bc}}$ & $4.67^{\mathrm{b}-\mathrm{d}}$ & $5.33^{\mathrm{d}}$ & $5.33^{\mathrm{a}-\mathrm{c}}$ & $25.00^{\mathrm{a}-\mathrm{e}}$ & $2.24^{\mathrm{c}-\mathrm{j}}$ \\
\hline 12 & SER 125 & $56.3^{\mathrm{a}}$ & $92.0^{\mathrm{a}}$ & $36.0^{\mathrm{bc}}$ & $5.67^{\mathrm{a}}$ & $6.33^{\mathrm{b}-\mathrm{d}}$ & $5.67^{\mathrm{a}-\mathrm{c}}$ & $19.00^{\mathrm{b}-\mathrm{g}}$ & $3.10^{\mathrm{a}-\mathrm{f}}$ \\
\hline 13 & Red Wolaita & $50.3^{a-g}$ & $86.7^{\mathcal{C}}$ & $44.0^{\mathrm{a}-\mathrm{c}}$ & $3.67^{\mathrm{e}-\mathrm{g}}$ & $7.67^{\mathrm{a}-\mathrm{d}}$ & $6.00^{a-c}$ & $21.33^{\mathrm{a}-\mathrm{g}}$ & $2.14^{\mathrm{c}-\mathrm{j}}$ \\
\hline 14 & Ramada & $46.7^{\mathrm{d}-\mathrm{h}}$ & $78.3^{\mathrm{d}-\mathrm{e}}$ & $38.3^{\mathrm{a}-\mathrm{c}}$ & $4.00^{\mathrm{d}-\mathrm{f}}$ & $6.67^{\mathrm{a}-\mathrm{d}}$ & $6.00^{a-c}$ & $16.70^{\mathrm{d}-\mathrm{g}}$ & $2.01^{\mathrm{d}-\mathrm{j}}$ \\
\hline 15 & Nasir & $48.7^{\mathrm{b}-\mathrm{h}}$ & $88.7^{\mathrm{a}-\mathrm{c}}$ & $56.3^{\mathrm{a}}$ & $4.67^{\mathrm{b}-\mathrm{d}}$ & $7.33^{\mathrm{a}-\mathrm{d}}$ & $7.33^{\mathrm{a}}$ & $16.33^{e-g}$ & $1.92^{\mathrm{e}-\mathrm{j}}$ \\
\hline 16 & SER 119 & $53.0^{\mathrm{a}-\mathrm{e}}$ & $90.7^{\mathrm{a}-\mathrm{c}}$ & $33.0^{\mathrm{bc}}$ & $5.67^{\mathrm{a}}$ & $7.33^{\mathrm{a}-\mathrm{d}}$ & $4.33^{\mathrm{c}}$ & $23.33^{\mathrm{a}-\mathrm{f}}$ & $2.74^{\mathrm{a}-\mathrm{i}}$ \\
\hline 17 & Dursitu & $54.3^{\mathrm{a}-\mathrm{c}}$ & $90.7^{\mathrm{a}-\mathrm{c}}$ & $36.3^{\mathrm{a}-\mathrm{c}}$ & $4.33^{\mathrm{c}-\mathrm{e}}$ & $7.33^{\mathrm{a}-\mathrm{d}}$ & $5.00^{\mathrm{bc}}$ & $20.33^{\mathrm{b}-\mathrm{g}}$ & $2.03^{\mathrm{d}-\mathrm{j}}$ \\
\hline 18 & Dimtu & $50.3^{\mathrm{a}-\mathrm{g}}$ & $90.0^{a-c}$ & $32.7^{\mathrm{bc}}$ & $5.00^{\mathrm{a}-\mathrm{c}}$ & $7.67^{a-d}$ & $4.67^{\mathrm{c}}$ & $15.67^{\mathrm{e}-\mathrm{g}}$ & $1.68^{\mathrm{f}-\mathrm{j}}$ \\
\hline 19 & $\begin{array}{l}\text { Awassa } \\
\text { Dume }\end{array}$ & $46.7^{\mathrm{d}-\mathrm{h}}$ & $75.0^{\mathrm{e}}$ & $37.0^{\mathrm{a}-\mathrm{c}}$ & $3.67^{\mathrm{e}-\mathrm{g}}$ & $7.00^{\mathrm{a}-\mathrm{d}}$ & $6.00^{a-c}$ & $15.00^{\mathrm{fg}}$ & $1.19^{\mathrm{j}}$ \\
\hline 20 & Ibado & $52.7^{\mathrm{a}-\mathrm{e}}$ & $88.7^{a-c}$ & $32.7^{\mathrm{bc}}$ & $4.67^{\mathrm{b}-\mathrm{d}}$ & $8.33^{\mathrm{ab}}$ & $4.67^{\mathrm{c}}$ & $24.33^{\mathrm{a}-\mathrm{f}}$ & $2.57^{b-j}$ \\
\hline 21 & Gobe Rashia & $54.3^{a-c}$ & $90.0^{\mathrm{a}-\mathrm{c}}$ & $29.3^{b c}$ & $5.00^{a-c}$ & $6.33^{\mathrm{b}-\mathrm{d}}$ & $4.67^{\mathcal{C}}$ & $27.67^{\mathrm{a}-\mathrm{c}}$ & $2.797^{\mathrm{a}-\mathrm{h}}$ \\
\hline 22 & Roba & $51.7^{\mathrm{a}-\mathrm{f}}$ & $91.3^{\mathrm{ab}}$ & $34.3^{\mathrm{bc}}$ & $4.00^{\mathrm{d}-\mathrm{f}}$ & $7.00^{\mathrm{a}-\mathrm{d}}$ & $5.33^{\mathrm{a}-\mathrm{c}}$ & $20.33^{b-g}$ & $3.06^{\mathrm{a}-\mathrm{f}}$ \\
\hline 23 & Brazil 2 & $45.3^{\mathrm{f}-\mathrm{h}}$ & $75.2^{\mathrm{e}}$ & $44.0^{\mathrm{ac}}$ & $3.67^{\mathrm{e}-\mathrm{g}}$ & $7.00^{\mathrm{a}-\mathrm{d}}$ & $6.00^{\mathrm{a}-\mathrm{c}}$ & $28.00^{\mathrm{a}-\mathrm{c}}$ & $2.14^{\mathrm{c}-\mathrm{j}}$ \\
\hline 24 & Awash 1 & $51.7^{\mathrm{a}-\mathrm{f}}$ & $81.7^{\mathrm{d}}$ & $39.7^{\mathrm{a}-\mathrm{c}}$ & $4.00^{\mathrm{d}-\mathrm{f}}$ & $7.33^{\mathrm{a}-\mathrm{d}}$ & $5.33^{a-c}$ & $16.00^{\mathrm{e}-\mathrm{g}}$ & $1.95^{\mathrm{e}-\mathrm{j}}$ \\
\hline 25 & Deme & $54.7^{\mathrm{ab}}$ & $92.0^{\mathrm{a}}$ & $49.0^{\mathrm{ab}}$ & $5.33^{\mathrm{ab}}$ & $8.33^{\mathrm{ab}}$ & $6.00^{a-c}$ & $30.33^{\mathrm{a}}$ & $4.18^{\mathrm{a}}$ \\
\hline 26 & SAB 632 & $43.0^{\mathrm{h}}$ & $75.0^{\mathrm{e}}$ & $28.3^{c}$ & $3.33^{\mathrm{fg}}$ & $6.33^{\mathrm{b}-\mathrm{d}}$ & $4.33^{c}$ & $25.00^{\mathrm{a}-\mathrm{e}}$ & $1.80^{f-j}$ \\
\hline 27 & Tatu & $44.3^{\mathrm{g}-\mathrm{h}}$ & $76.7^{\mathrm{e}}$ & $37.0^{\mathrm{a}-\mathrm{c}}$ & $3.00^{\mathrm{g}}$ & $6.00^{\mathrm{b}-\mathrm{d}}$ & $6.00^{a-c}$ & $18.33^{\mathrm{c}-\mathrm{g}}$ & $1.32^{\mathrm{h}-\mathrm{j}}$ \\
\hline 28 & ALB 179 & $51.3^{\mathrm{a}-\mathrm{f}}$ & $89.3^{\mathrm{a}-\mathrm{c}}$ & $32.3^{b c}$ & $4.33^{\mathrm{c}-\mathrm{e}}$ & $6.00^{\mathrm{b}-\mathrm{d}}$ & $6.00^{\mathrm{a}-\mathrm{c}}$ & $26.33^{\mathrm{a}-\mathrm{d}}$ & $3.58^{\mathrm{a}-\mathrm{c}}$ \\
\hline 29 & ALB 209 & $51.3^{\mathrm{a}-\mathrm{f}}$ & $88.3^{\mathrm{a}-\mathrm{c}}$ & $29.0^{b c}$ & $4.00^{\mathrm{d}-\mathrm{f}}$ & $8.00^{\mathrm{a}-\mathrm{c}}$ & $4.11^{\mathrm{c}}$ & $20.33^{b-g}$ & $2.29^{b-j}$ \\
\hline 30 & ALB 61 & $51.3^{\mathrm{a}-\mathrm{f}}$ & $88.3^{\mathrm{a}-\mathrm{c}}$ & $29.7^{\mathrm{bc}}$ & $4.33^{\mathrm{c}-\mathrm{e}}$ & $9.00^{\mathrm{a}}$ & $4.00^{c}$ & $15.00^{\mathrm{fg}}$ & $1.86^{\mathrm{e}-\mathrm{j}}$ \\
\hline 31 & ALB 25 & $53.3^{\mathrm{a}-\mathrm{d}}$ & $89.7^{\mathrm{a}-\mathrm{c}}$ & $32.3^{b c}$ & $4.33^{\mathrm{c}-\mathrm{e}}$ & $7.33^{\mathrm{a}-\mathrm{d}}$ & $4.33^{\mathrm{c}}$ & $21.67^{\mathrm{a}-\mathrm{g}}$ & $2.74^{\mathrm{a}-\mathrm{i}}$ \\
\hline 32 & NVA 682 & $43.7^{\mathrm{g}-\mathrm{h}}$ & $76.7^{\mathrm{e}}$ & $25.7^{\mathcal{C}}$ & $4.00^{\mathrm{d}-\mathrm{f}}$ & $6.00^{\mathrm{b}-\mathrm{d}}$ & $4.33^{c}$ & $26.67^{\mathrm{a}-\mathrm{c}}$ & $3.54^{\mathrm{a}-\mathrm{d}}$ \\
\hline \multirow[t]{3}{*}{33} & NVA 517 & $53.7^{\mathrm{a}-\mathrm{c}}$ & $88.3^{\mathrm{a}-\mathrm{c}}$ & $44.7^{\mathrm{a}-\mathrm{c}}$ & $4.67^{\mathrm{b}-\mathrm{d}}$ & $8.00^{a-c}$ & $5.67^{a-c}$ & $28.67^{\mathrm{ab}}$ & $3.81^{\mathrm{ab}}$ \\
\hline & LSD & 6.7 & 4.3 & 20.10 & 0.87 & 2.33 & 2.26 & 9.98 & 1.50 \\
\hline & $\mathrm{CV}$ & 8.33 & 3.12 & 34.8 & 12.4 & 20.9 & 26.1 & 29.6 & 40.6 \\
\hline
\end{tabular}

characters plant height and biological yield. This implies greater influence of environmental factors for the phenotypic expression of these characters. Relatively medium phenotypic variance $(50-100 \%)$ observed for days to maturity whereas. relatively lower phenotypic variance was recorded for days to flowering, number of nodes, stem diameter, inter node length, leaf area, LAI, pods per plant, seeds per pod and HI. On the other hand, genotypic variance ranged from 0.01 to 763.32 with higher genotypic variance for biological yield and HSW. Moderate genotypic $\left(\sigma_{\mathrm{g}}^{2}\right)$ variance were observed for days to maturity and grain yield. Lower genotypic variance $\left(\sigma_{\mathrm{g}}^{2}\right)$ was observed for plant height, stem diameter, number of node on the main stem, internode length, LAI, biological yield, seeds per pod. Singh [13] reported that genotypic variance was $\left(\sigma_{\mathrm{g}}^{2}\right)$ variable for different agronomic traits of different common bean genotypes.

In general phenotypic coefficient of variation (PCV) varied from 8.55 for days to maturity to 48.02 for LAI Table 3 . According to Sivasubramanian and Madhavamenon [22] PCV grouped as high if PCV $>20 \%$, moderate if PCV is $10-20 \%$ and low if PCV is below $10 \%$. Based on this grouping, traits such as plant height, number of nodes, internode length, leaf area, LAI, biological yield, pods per plant, HI and HSW had higher PCV. Conversely, traits like days to flowering stem diameter and seeds per pod exhibited moderate PCV where as only days to maturity showed lower PCV with PCV value below $10 \%$. This reflected the pronounced influence of environmental factors for the expression of these characters. This 
TABLE 2: Mean performance of genotypes of common bean for yield and yield components.

\begin{tabular}{|c|c|c|c|c|c|c|c|}
\hline SN & Genotype & Pods per plant & Seeds per pod & $\mathrm{HSW}(\mathrm{g})$ & Biological yield (kg/ha) & Grain yield (kg/ha) & $\mathrm{HI}$ \\
\hline 1 & SEC 20 & $22.67^{\mathrm{a}}$ & $5.72^{\mathrm{a}}$ & $16.33^{\mathrm{n}-\mathrm{q}}$ & $17670^{\mathrm{a}}$ & $4462^{\mathrm{a}}$ & $0.12^{f-j}$ \\
\hline 2 & SMC 22 & $8.33^{\mathrm{i}-\mathrm{n}}$ & $4.33^{\mathrm{c}-\mathrm{e}}$ & $19.67^{1-n}$ & $7667^{\mathrm{j}-\mathrm{m}}$ & $1938^{\mathrm{pq}}$ & $0.12^{\mathrm{f}-\mathrm{j}}$ \\
\hline 3 & SMC 21 & $8.33^{\mathrm{i}-\mathrm{n}}$ & $4.70^{\mathrm{b}-\mathrm{d}}$ & $19.33^{\mathrm{mn}}$ & $9000^{\mathrm{g}-\mathrm{k}}$ & $2355^{\mathrm{m}-\mathrm{o}}$ & $0.12^{\mathrm{d}-\mathrm{h}}$ \\
\hline 4 & SEC 23 & $18.00^{\mathrm{b}}$ & $5.33^{\mathrm{ab}}$ & $15.33^{\mathrm{pq}}$ & $15000^{\mathrm{a}-\mathrm{d}}$ & $3718^{\mathrm{c}}$ & $0.11^{\mathrm{g}-\mathrm{j}}$ \\
\hline 5 & ALB 204 & $15.33^{\mathrm{b}-\mathrm{d}}$ & $4.70^{\mathrm{b}-\mathrm{d}}$ & $23.00^{\mathrm{j}-1}$ & $11670^{\mathrm{d}-\mathrm{i}}$ & $3545^{c-e}$ & $0.17^{\mathrm{a}}$ \\
\hline 6 & Waji & $11.00^{e-j}$ & $4.33^{c-e}$ & $28.00^{\mathrm{hi}}$ & $12000^{\mathrm{c}-\mathrm{h}}$ & $3430^{\mathrm{d}-\mathrm{f}}$ & $0.13^{c-f}$ \\
\hline 7 & Brazil 1 & $9.33^{\mathrm{g}-\mathrm{n}}$ & $3.70^{\mathrm{e}-\mathrm{g}}$ & $34.67^{\mathrm{ef}}$ & $9333^{\mathrm{g}-\mathrm{k}}$ & $2587^{\operatorname{lm}}$ & $0.13^{\mathrm{c}-\mathrm{e}}$ \\
\hline 8 & SAB-735 & $7.67^{j-n}$ & $3.01^{\mathrm{g}}$ & $31.00^{\mathrm{gh}}$ & $6667^{\mathrm{k}-\mathrm{m}}$ & $1775^{\mathrm{q}}$ & $0.12^{\mathrm{d}-\mathrm{h}}$ \\
\hline 9 & Awash Melka & $7.33^{j-n}$ & $4.33^{\mathrm{c}-\mathrm{e}}$ & $13.67^{\mathrm{q}}$ & $5000^{\operatorname{lm}}$ & $1147^{\mathrm{s}}$ & $0.11^{\mathrm{ij}}$ \\
\hline 10 & Awash 2 & $10.67^{f-k}$ & $4.70^{\mathrm{b}-\mathrm{d}}$ & $15.00^{\mathrm{pq}}$ & $8000^{\mathrm{i}-\mathrm{m}}$ & $1833^{\mathrm{q}}$ & $0.11^{\mathrm{h}-\mathrm{j}}$ \\
\hline 11 & Melka Dima & $9.00^{\mathrm{h}-\mathrm{n}}$ & $4.00^{\mathrm{d}-\mathrm{f}}$ & $39.00^{\mathrm{d}}$ & $12330^{c-j}$ & $3642^{\mathrm{cd}}$ & $0.14^{\mathrm{b}-\mathrm{d}}$ \\
\hline 12 & SER 125 & $14.67^{\mathrm{b}-\mathrm{f}}$ & $5.00^{\mathrm{a}-\mathrm{c}}$ & $23.67^{\mathrm{jk}}$ & $16670^{\mathrm{ab}}$ & $4235^{\mathrm{ab}}$ & $0.12^{\mathrm{f}-\mathrm{j}}$ \\
\hline 13 & Red Wolaita & $10.67^{\mathrm{f}-\mathrm{k}}$ & $4.70^{\mathrm{b}-\mathrm{d}}$ & $19.33^{\mathrm{mn}}$ & $9333^{g-k}$ & $2310^{\text {no }}$ & $0.12^{f-j}$ \\
\hline 14 & Ramada & $9.00^{\mathrm{h}-\mathrm{n}}$ & $4.00^{\mathrm{d}-\mathrm{f}}$ & $37.67^{\mathrm{de}}$ & $13330^{\mathrm{b}-\mathrm{f}}$ & $3017^{h-j}$ & $0.10^{j}$ \\
\hline 15 & Nasir & $13.33^{\mathrm{c}-\mathrm{g}}$ & $5.33^{\mathrm{ab}}$ & $19.33^{\mathrm{mn}}$ & $11670^{\mathrm{d}-\mathrm{i}}$ & $3343^{\mathrm{e}-\mathrm{g}}$ & $0.13^{\mathrm{c}-\mathrm{f}}$ \\
\hline 16 & SER 119 & $14.67^{\mathrm{b}-\mathrm{f}}$ & $5.00^{a-c}$ & $26.00^{\mathrm{ij}}$ & $15670^{a-c}$ & $4238^{\mathrm{ab}}$ & $0.12^{\mathrm{d}-\mathrm{h}}$ \\
\hline 17 & Dursitu & $11.33^{\mathrm{d}-\mathrm{j}}$ & $4.70^{\mathrm{b}-\mathrm{d}}$ & $19.00^{\mathrm{m}-\mathrm{o}}$ & $11330^{\mathrm{d}-\mathrm{f}}$ & $4113^{\mathrm{b}}$ & $0.12^{\mathrm{d}-\mathrm{h}}$ \\
\hline 18 & Dimtu & $12.00^{\mathrm{c}-\mathrm{i}}$ & $5.00^{\mathrm{a}-\mathrm{c}}$ & $18.33^{\mathrm{m}-\mathrm{p}}$ & $11330^{\mathrm{d}-\mathrm{f}}$ & $2832^{i-k}$ & $0.12^{\mathrm{e}-\mathrm{i}}$ \\
\hline 19 & Awassa Dume & $13.00^{\mathrm{c}-\mathrm{h}}$ & $5.00^{\mathrm{a}-\mathrm{c}}$ & $19.67^{1-m}$ & $10000^{\mathrm{f}-\mathrm{k}}$ & 3058. & $0.14^{\mathrm{bc}}$ \\
\hline 20 & Ibado & $6.33^{1-n}$ & $3.33^{\mathrm{fg}}$ & $45.67^{\mathrm{ab}}$ & $11000^{e-j}$ & $3235^{\mathrm{f}-\mathrm{h}}$ & $0.13^{\mathrm{c}-\mathrm{e}}$ \\
\hline 21 & Gobe Rashia & $7.33^{\mathrm{j}-\mathrm{n}}$ & $4.33^{\mathrm{c}-\mathrm{e}}$ & $43.00^{\mathrm{bc}}$ & $12000^{\mathrm{c}-\mathrm{h}}$ & $3370^{e-g}$ & $0.13^{\mathrm{c}-\mathrm{e}}$ \\
\hline 22 & Roba & $10.00^{\mathrm{g}-\mathrm{m}}$ & $5.70^{\mathrm{a}}$ & $15.67^{\mathrm{o}-\mathrm{q}}$ & $7667^{\mathrm{j}-\mathrm{m}}$ & $2145^{\text {op }}$ & $0.13^{\mathrm{c}-\mathrm{e}}$ \\
\hline 23 & Brazil 2 & $10.33^{\mathrm{g}-1}$ & $4.00^{\mathrm{d}-\mathrm{f}}$ & $33.00^{\mathrm{fg}}$ & $10670^{f-j}$ & $2828^{\mathrm{i}-\mathrm{k}}$ & $0.12^{\mathrm{d}-\mathrm{h}}$ \\
\hline 24 & Awash 1 & $13.33^{\mathrm{c}-\mathrm{g}}$ & $4.00^{\mathrm{d}-\mathrm{f}}$ & $16.67^{\mathrm{n}-\mathrm{q}}$ & $9333^{\mathrm{g}-\mathrm{k}}$ & $2813^{\mathrm{j}-1}$ & $0.12^{\mathrm{e}-\mathrm{i}}$ \\
\hline 25 & Deme & $6.00^{\mathrm{mn}}$ & $4.00^{\mathrm{d}-\mathrm{f}}$ & $48.67^{\mathrm{a}}$ & $14670^{\mathrm{a}-\mathrm{e}}$ & $3715^{\mathrm{c}}$ & $0.12^{f-j}$ \\
\hline 26 & SAB 632 & $6.67^{\mathrm{k}-\mathrm{n}}$ & $3.70^{\mathrm{e}-\mathrm{g}}$ & $38.00^{\mathrm{de}}$ & $8333^{\text {h-l }}$ & $2220^{\text {no }}$ & $0.13^{\mathrm{c}-\mathrm{g}}$ \\
\hline 27 & Tatu & $5.33^{\mathrm{n}}$ & $3.00^{\mathrm{g}}$ & $30.00^{\mathrm{gh}}$ & $4333^{\mathrm{m}}$ & $1475^{\mathrm{r}}$ & $0.15^{\mathrm{b}}$ \\
\hline 28 & ALB 179 & $14.67^{\mathrm{b}-\mathrm{f}}$ & $4.70^{\mathrm{b}-\mathrm{d}}$ & $23.67^{\mathrm{jk}}$ & $12000^{c-h}$ & $3312^{\mathrm{e}-\mathrm{g}}$ & $0.13^{\mathrm{c}-\mathrm{f}}$ \\
\hline 29 & ALB 209 & $15.00^{\mathrm{b}-\mathrm{e}}$ & $4.70^{\mathrm{b}-\mathrm{d}}$ & $23.00^{\mathrm{j}-1}$ & $12330^{\mathrm{c}-\mathrm{g}}$ & $3413^{\mathrm{d}-\mathrm{f}}$ & $0.13^{\mathrm{c}-\mathrm{f}}$ \\
\hline 30 & ALB 61 & $15.00^{\mathrm{b}-\mathrm{e}}$ & $4.00^{\mathrm{d}-\mathrm{f}}$ & $20.33^{\mathrm{k}-\mathrm{m}}$ & $11330^{d-j}$ & $2710^{\mathrm{kl}}$ & $0.13^{\mathrm{c}-\mathrm{g}}$ \\
\hline 31 & ALB 25 & $15.67^{b c}$ & $3.70^{\mathrm{e}-\mathrm{f}}$ & $21.00^{\mathrm{k}-\mathrm{m}}$ & $12330^{\mathrm{f}-\mathrm{k}}$ & $3172^{\mathrm{gh}}$ & $0.13^{\mathrm{c}-\mathrm{g}}$ \\
\hline 32 & NVA 682 & $6.33^{1-m}$ & $3.33^{\mathrm{fg}}$ & $40.67^{\mathrm{cd}}$ & $10330^{\mathrm{b}-\mathrm{f}}$ & $2453^{\mathrm{mn}}$ & $0.11^{\mathrm{g}-\mathrm{j}}$ \\
\hline \multirow[t]{3}{*}{33} & NVA 517 & $9.67^{\mathrm{g}-\mathrm{m}}$ & $3.70^{e-f}$ & $39.00^{\mathrm{d}}$ & $13330^{\mathrm{b}-\mathrm{f}}$ & $4325^{\mathrm{ab}}$ & $0.14^{\mathrm{bc}}$ \\
\hline & LSD & 4.0 & 0.97 & 3.61 & 3717 & 239 & 0.01 \\
\hline & $\mathrm{CV}$ & 22.41 & 13.8 & 8.3 & 20.7 & 15.49 & 13.04 \\
\hline
\end{tabular}

finding is in agreement with the result of Kassaye [7] that reported higher PCV for plant height, number of nodes on main stem, pods per plant, internode length and HSW. Generally in common bean, a wide range of GCV and PCV values were reported by [23-25]. Moreover, Berhanu [26], Kumar et al. [27], and Baranwal et al. [28] were also reported moderate PCV for grain yield, TSW and tillers per plant for wheat cultivars. In line with this, genotypic coefficient variance (GCV) varied from $1.88 \%$ to $37.72 \%$ (Table 3 ). The lowest GCV $(1.88 \%)$ was recorded for inter node length while the highest GCV (37.72\%) for HSW. As this investigation indicated that higher GCV (>20\%) was observed for LAI, biological yield, pods per plant, HSW and grain yield whereas moderate GCV (10-20\%) was recorded for stem diameter, leaf area and seeds per pod. This finding disagrees with the result of Ejigu et al. [29] who reported low GCV for number of pods per plant and HSW. On the other hand, lower GCV $(<10 \%)$ was seen for days to flowering, days to maturity, plant height, number of nodes on main stem, internode length and HI. This finding is in agreement with the result of Ejigu et al. [29] that reported low GCV for days to flowering and days to maturity. Singh [13] reported that there was wider genotypic variability with respect to agronomic traits in different common bean genotypes.

3.4.2. Heritability and Genetic Advance. In general heritability in broad sense $\left(\mathrm{H}^{2}\right)$ ranged from $0.52 \%$ for internode length which was the lowest to $95.33 \%$ for HSW which was the highest value (Table 3). Johnson et al. [30] classified heritability estimates as low $(<30 \%)$, moderate $(30-60 \%)$ and high $(>60 \%)$. Based on this classification, days to maturity, pods per plant, HSW and grain yield exhibited high $H^{2}$ estimates indicating that environment has low influence for the expression of the characters which suggests direct 
TABLE 3: Phenotypic and genotypic coefficient of variability, heritability and genetic advance for genotypes.

\begin{tabular}{|c|c|c|c|c|c|c|c|}
\hline Trait & $\sigma_{\mathrm{p}}^{2}$ & $\sigma_{\mathrm{p}}^{2}$ & $\sigma_{\mathrm{e}}^{2}$ & PCV & $\mathrm{GCV}$ & $\mathrm{H}(\%)$ & GA (\%) \\
\hline Days to flowering & 28.92 & 11.58 & 17.34 & 10.76 & 6.80 & 40.00 & 10.37 \\
\hline Days to maturity & 52.66 & 45.66 & 7.00 & 8.55 & 7.96 & 86.70 & 16.15 \\
\hline Plant height & 149.55 & -2.24 & 147.31 & 34.57 & -4.24 & -1.50 & -2.23 \\
\hline Number of nodes & 2.20 & 0.15 & 2.05 & 21.72 & 5.71 & 6.90 & 2.69 \\
\hline Stem diameter & 0.67 & 0.38 & 0.29 & 18.93 & 14.30 & 56.90 & 26.54 \\
\hline Inter node length & 1.91 & 0.01 & 1.90 & 26.00 & -1.88 & -0.52 & -2.85 \\
\hline Leaf area & 47.43 & 9.98 & 37.45 & 33.30 & 15.30 & 21.00 & 15.53 \\
\hline LAI & 1.23 & 0.35 & 0.88 & 48.02 & 25.56 & 28.33 & 27.62 \\
\hline Biological yield & 1282.70 & 763.32 & 519.38 & 32.53 & 25.10 & 59.50 & 40.04 \\
\hline Pods per plant & 19.76 & 13.51 & 6.25 & 39.86 & 32.96 & 68.40 & 53.20 \\
\hline Seeds per pod & 0.74 & 0.38 & 0.36 & 19.85 & 14.25 & 51.55 & 12.59 \\
\hline Harvest index & 0.05 & 0.01 & 0.04 & 38.63 & 7.73 & 20.00 & 26.37 \\
\hline HSW & 105.25 & 100.34 & 4.91 & 38.63 & 37.72 & 95.33 & 74.52 \\
\hline Grain yield & 87.29 & 65.79 & 21.50 & 31.20 & 27.10 & 75.40 & 46.95 \\
\hline
\end{tabular}

selection using these characters as major contributors of yield components to improve yield of common bean genotypes. This high heritability may be due to additive gene effects hence these traits are likely to respond to direct selection in order to improve common bean grain yield through direct selection for grain yield related traits. Relatively moderate $H^{2}$ was recorded for traits days to flowering, stem diameter, biological yield and seeds per pod which may be occurred due to influence of the environment on the polygenic nature of these traits. On the other hand, $\mathrm{H}^{2}$ was low for traits plant height, number of nodes on main stem, internode length, leaf area, LAI and $\mathrm{HI}$ that limits the possibility of including the traits in order to select desirable genotypes. This may be due to the higher influence of environment for the expression of phenotypic variation than genotypic variation.

Genetic advance as a percent mean was ranged from $2.23 \%$ for plant height to $74.52 \%$ for HSW Table 3 . This result indicated that selecting the top $5 \%$ of the genotypes could result in an advance of $2.23-74.52 \%$ over the respective population mean. As suggested by Sivasubramanian and Madhavamenon [22], genetic advance as percent of mean was classified as low $(<10 \%)$, moderate $(10-20 \%)$ and high $(>20 \%)$. Based on this classification, traits like stem diameter, LAI, biological yield, pods per plant, HI, HSW and grain yield exhibited high genetic advance. Traits days to flowering, days to maturity, leaf area and seeds per pod attained moderate genetic advance. In contrast, plant height, number of nodes on main stem and internode length had low genetic advance. Trait like number of pods per plant, HSW and grain yield exhibited high heritability coupled with high genetic advance while stem diameter and biological yield showed moderate heritability coupled with high genetic advance. Hence, these traits should be given top priority during selection in common bean breeding because they are the major portion of genetic variation attributable to additive gene action and selection may be effective in early generations for these traits. In line with this, days to flowering and seeds per pod associated with moderate heritability with moderate genetic advance. Moderate heritability accompanied with moderate genetic advance as percent of mean was recorded by days to flowering and seeds per pod. Both additive and nonadditive gene actions are involved in the expression of these traits. This result agreed with the findings of Alemayehu [23] that reported moderate estimates of $\mathrm{H}^{2}$ coupled with moderate genetic advance for number of seeds per pod in common bean.

3.4.3. Phenotypic and Genotypic Correlations. The data for phenotypic and genotypic correlation of genotypes are depicted in Table 4. Genotypic correlation coefficient values ranged from -0.74 to 0.99 . Stem diameter $\left(r_{\mathrm{g}}=0.84\right)$, LAI $\left(r_{\mathrm{g}}=0.74\right)$, biological yield $\left(r_{\mathrm{g}}=0.96\right)$, pods per plant $\left(r_{\mathrm{g}}=0.56\right)$, seeds per pod $\left(r_{\mathrm{g}}=0.34\right)$, days to flowering $\left(r_{\mathrm{g}}=0.73\right)$ and days to maturity $\left(r_{\mathrm{g}}=0.59\right)$ exhibited significant and positive genotypic association with grain yield where the highest genotypic correlation coefficient was recorded for biological yield. This indicates that the genotypes with high leaf area index, vegetative yield, pods per plant and seeds per pod were producing higher grain yield. In addition to this the late flowering and maturing type of genotypes were also producing higher yield rather than early flowering and maturity type. This result was similar with the findings of Kifle et al. [31], Kumar et al. [32], and Ebrahimnejad and Rameeh [33] who reported the positive and significant association of grain yield with biological yield. Sadeghi et al. [34] reported highly significant correlations of seed yield with seed number per plant, seed number per pod, pod number per plant, days to flowering and HSW. In line with this, stem diameter $\left(r_{\mathrm{g}}=0.93\right)$, LAI $\left(r_{\mathrm{g}}=0.80\right)$, pods per plant $\left(r_{\mathrm{g}}=0.61\right)$, seeds per pod $\left(r_{\mathrm{g}}=0.38\right)$, days to flowering $\left(r_{\mathrm{g}}=0.78\right)$ and days to maturity $\left(r_{\mathrm{g}}=0.61\right)$ showed significant positive genotypic correlation with biological yield Table 4 . Similarly, stem diameter with LAI $\left(r_{\mathrm{g}}=0.84\right)$, pods per plant $\left(r_{\mathrm{g}}=0.59\right)$, seeds per pod $\left(r_{\mathrm{g}}=0.55\right)$, days to flowering $\left(r_{\mathrm{g}}=0.99\right)$ and days to maturity $\left(r_{\mathrm{g}}=0.79\right)$ had significant positive association.

In general phenotypic correlation coefficients for agronomic traits ranged from -0.62 to 0.93 Table 4 . Stem diameter $\left(r_{\mathrm{p}}=0.74\right)$, LAI $\left(r_{\mathrm{p}}=0.52\right)$, days to flowering $\left(r_{\mathrm{p}}=0.58\right)$, days to maturity $\left(r_{\mathrm{p}}=0.55\right)$, biological yield $\left(r_{\mathrm{p}}=0.93\right)$ and pods per plant 
TABLE 4: Genotypic (above diagonal) and phenotypic (below diagonal) correlation coefficients for agronomic traits of common bean genotypes.

\begin{tabular}{lccccccccccc}
\hline & SD & LA & LAI & BY & PPP & SPP & HSW & HI & GY & DF & DM \\
\hline SD & & $0.09^{\mathrm{NS}}$ & $0.84^{*}$ & $0.93^{*}$ & $0.49^{*}$ & $0.55^{*}$ & $0.01^{\mathrm{NS}}$ & $-0.24^{\mathrm{NS}}$ & $0.84^{*}$ & $0.99^{*}$ & $0.79^{*}$ \\
LA & $0.15^{\mathrm{NS}}$ & & $0.69^{*}$ & $0.18^{\mathrm{NS}}$ & $-0.58^{*}$ & $-0.82^{*}$ & $0.99^{*}$ & $0.36^{*}$ & $0.27^{\mathrm{NS}}$ & $0.11^{\mathrm{NS}}$ & $0.25^{\mathrm{NS}}$ \\
LAI & $0.54^{*}$ & $0.72^{*}$ & & $0.80^{*}$ & $0.21^{\mathrm{NS}}$ & $0.09^{\mathrm{NS}}$ & $0.38^{*}$ & $0.08^{\mathrm{NS}}$ & $0.74^{*}$ & $0.94^{*}$ & $0.81^{*}$ \\
BY & $0.82^{*}$ & $0.24^{\mathrm{NS}}$ & $0.56^{*}$ & & $0.61^{*}$ & $0.38^{*}$ & $0.14^{\mathrm{NS}}$ & $-0.08^{\mathrm{NS}}$ & $0.96^{*}$ & $0.78^{*}$ & $0.61^{*}$ \\
PPP & $0.39^{*}$ & $-0.33^{*}$ & $0.08^{\mathrm{NS}}$ & $0.53^{*}$ & & $0.74^{*}$ & $-0.66^{*}$ & $0.08^{\mathrm{NS}}$ & $0.56^{*}$ & $0.67^{*}$ & $0.51^{*}$ \\
SPP & $0.45^{*}$ & $-0.33^{*}$ & $0.07^{\mathrm{NS}}$ & $0.32^{*}$ & $0.59^{*}$ & & $-0.74^{*}$ & $-0.13^{\mathrm{NS}}$ & $0.34^{*}$ & $0.63^{*}$ & $0.58^{*}$ \\
HSW & $0.07^{\mathrm{NS}}$ & $0.70^{*}$ & $0.37^{*}$ & $0.23^{\mathrm{NS}}$ & $-0.59^{*}$ & $-0.62^{*}$ & & $0.21^{\mathrm{NS}}$ & $0.16^{\mathrm{NS}}$ & $-0.27^{\mathrm{NS}}$ & $-0.21^{\mathrm{NS}}$ \\
HI & $-0.13^{\mathrm{NS}}$ & $0.18^{\mathrm{NS}}$ & $0.07^{\mathrm{NS}}$ & $-0.06^{\mathrm{NS}}$ & $0.10^{\mathrm{NS}}$ & $-0.08^{\mathrm{NS}}$ & $0.13^{\mathrm{NS}}$ & & $0.23^{\mathrm{NS}}$ & $0.07^{\mathrm{NS}}$ & $0.09^{\mathrm{NS}}$ \\
GY & $0.74^{*}$ & $0.29^{\mathrm{NS}}$ & $0.52^{*}$ & $0.93^{*}$ & $0.49^{*}$ & $0.28^{\mathrm{NS}}$ & $0.23^{\mathrm{NS}}$ & $0.19^{\mathrm{NS}}$ & & $0.73^{*}$ & $0.59^{*}$ \\
DF & $0.72^{*}$ & $0.19^{\mathrm{NS}}$ & $0.58^{*}$ & $0.57^{*}$ & $0.44^{*}$ & $0.44^{*}$ & $-0.16^{\mathrm{NS}}$ & $0.02^{\mathrm{NS}}$ & $0.58^{*}$ & & $0.99^{*}$ \\
DM & $0.71^{*}$ & $0.21^{\mathrm{NS}}$ & $0.59^{*}$ & $0.54^{*}$ & $0.47^{*}$ & $0.49^{*}$ & $-0.17^{\mathrm{NS}}$ & $0.09^{\mathrm{NS}}$ & $0.55^{*}$ & $0.92^{*}$ & \\
\hline
\end{tabular}

* indicates significant differences at $5 \%$ level and 'ns' indicates non-significance.

$\left(r_{\mathrm{p}}=0.49\right)$ had significant positive correlation with grain yield where the highest phenotypic correlation coefficient was recorded between grain yield and biological yield. The significant and positive correlation of these traits with grain yield was probably due to more photosynthetic product partitioned to grain that increased their weight [35]. Biological yield showed significant positive associations with stem diameter $\left(r_{\mathrm{p}}=0.82\right)$, LAI $\left(r_{\mathrm{p}}=0.56\right)$, pods per plant $\left(r_{\mathrm{p}}=0.53\right)$, seeds per pod $\left(r_{\mathrm{p}}=0.32\right)$, days to flowering $\left(r_{\mathrm{p}}=0.57\right)$ and days to maturity $\left(r_{\mathrm{p}}=0.54\right)$. Moreover, significant positive association were observed between LAI with stem diameter $\left(r_{\mathrm{p}}=0.54\right)$, HSW $\left(r_{\mathrm{p}}=0.37\right)$, leaf area $\left(r_{\mathrm{p}}=0.72\right)$, days to flowering $\left(r_{\mathrm{p}}=0.58\right)$ and days to maturity $\left(r_{\mathrm{p}}=0.59\right)$. Similarly significant positive association were observed between pods per plant with stem diameter $\left(r_{\mathrm{p}}=0.39\right)$, seeds per pod $\left(r_{\mathrm{p}}=0.59\right)$, days to flowering $\left(r_{\mathrm{p}}=0.44\right)$ and days to maturity $\left(r_{\mathrm{p}}=0.47\right)$. Stem diameter was revealed significant positive association with seeds per pod $\left(r_{\mathrm{p}}=0.45\right)$, days to flowering $\left(r_{\mathrm{p}}=0.72\right)$ and days to maturity $\left(r_{\mathrm{p}}=0.71\right)$. In addition seeds per pod with days to flowering $\left(r_{\mathrm{p}}=0.44\right)$ and days to maturity $\left(r_{\mathrm{p}}=0.49\right)$, leaf area with HSW $\left(r_{\mathrm{p}}=0.70\right)$ and days to flowering with days to maturity $\left(r_{\mathrm{p}}=0.92\right)$ were revealed significant positive association.

\section{Concussion}

Genetic variability is a measure of the tendency of individual genotypes in a population to vary from one another for certain characters of interest under consideration which could arise due to a number of factors. Understanding these variability, heritability and association between grain yield and other agronomic traits is necessary in plant breeding, especially for the individual plant selection. Analysis of variance revealed that genotypes of common bean significantly differed for yield components and yield. Thus, testing of common bean genotypes is among the best technologies to improve productivity and for specific area recommendation. Results of this experiment showed that genotype SEC 20 gave the highest grain yield. With respect to diseases reactions, genotypes SEC 20, SMC 22, ALB 204, Waji, Awash Melka,
Awash 2, SER 125, Ramada, SER 119, Dimtu, Awassa Dume, Roba, ALB 179 and ALB 61 found to be resistant to angular leaf spot. In line with this, genotypes Waji, Awash Melka, Awash 2, Melka Dima, SER 125, Red Wolaita, Nasir and Gobe Rashia were not totally attacked by bean rust exhibiting best resistant reaction. Phenotypic coefficient of variation (PCV) varied from 8.55 for days to maturity to 48.02 for LAI where traits plant height, number of nodes, internode length, leaf area, LAI, biological yield, pods per plant, HI and HSW had higher PCV. Genotypic coefficient variance (GCV) varied from $1.88 \%$ to $37.72 \%$ with the highest GCV recorded for HSW. Heritability in broad sense $\left(H^{2}\right)$ ranged from $0.52 \%$ to 95.33\% with the greatest observed for HSW. Genetic advance as a percent mean was ranged from $2.23 \%$ for plant height to $74.52 \%$ for HSW. The present study revealed significant variation among genotypes for traits considered except few insignificant traits. In addition, almost all the genotypes were well adapted to the study area and hence, the high yielding genotypes could be directly used as seed sources for production of common bean and genotype with best diseases reaction with high heritability can possibility used in common bean improvement program in breeding.

\section{Data Availability}

The data used to support the findings of this study are available from the corresponding author upon request.

\section{Conflict of Interest}

The authors declare that there is no conflict of interest regarding publication of this manuscript.

\section{Acknowledgments}

The first author thanks Wolaita sodo University for sponsoring his postgraduate study scholarship and Areka research center for hosting the field experiment. We thank Melkassa 
agricultural research center for providing seeds of haricot bean genotypes.

\section{References}

[1] J. Broughton, G. Hernandez, M. Blair, S. Beebe, P. Gepts, and J. Vanderleyden, "Beans (Phaseolus spp.) - model food legumes," Plant and Soil, vol. 252, no. 1, pp. 55-128, 2003.

[2] A. Asfaw, W. Blair, and C. Almekinders, "Genetic diversity and population structure of common bean (Phaseolus vulgaris L.) landraces from the East African highlands," Theoretical and Applied Genetics, vol. 120, no. 1, pp. 1-12, 2009.

[3] N. Dereje, G. Teshome, and A. Amare, Ed., "Low land pulses improvement in Ethiopia," Twenty-five Years of Research Experience in Low Land Crops Proc. of the 25th Anniversary of Naz. R.C. 22-23, pp. 41-47, 1995.

[4] U. Singh and B. Singh, "Tropical grain legumes as important human foods," Economic Botony, vol. 46, no. 3, pp. 310-321, 1992.

[5] S. Akibode and M. Maredia, Global and regional trends in production, trade and consumption of food legume crops, 2011, Rep.Sub. to SPIA Mar. 27.

[6] Central agricultural census commission (CACC), "Ethiopian agricultural sample Enumeration, 2001/2002: Report on the Primary Result of Area, Production and Yield of Temporary Crops (Meher Season Private Peasant Holding) Part 1. A. A," Central Statistical Authority, Addis Ababa, 2002.

[7] N. Kassaye, "Studies on genetic divergence in common bean (Phaseolus vulgaris L.) introductions of Ethiopia," M. S. Thesis, A.A.U., Addis Ababa, 2006.

[8] O. Humpreys, "A genetic approach to the multivariate differentiation perennial ryegrass (Loliumperenne L.) cultivars," Heredity, vol. 66, no. 3, pp. 437-443, 1991.

[9] J. Newbury and V. Ford-Lloyd, "Estimation of genetic diversity," in Plant Genetic Conservation: The In Situ Approach, N. Maxted, Ed., pp. 192-206, Chapman and Hall, London, 1997.

[10] E. Abayneh, Soils of Areka Agriculture Research Center, p. 77, 2003, Technical Paper No Unpublished material.

[11] N. Rajeshwar, R. S. Vaishnav, K. Srivastava, and K. K. Issar, "Genetic variability, heritability and genetic advance for yield components in winged bean (Psophocarpus tetragonalobus L.)," Asian Journal of Biological Sciences, vol. 4, no. 2, pp. 298-299, 2010.

[12] W. Burton and H. Devane, "Estimating heritability in tall Fescue (Festuca arundinacea) from replicated clonal materials," Agronomy Journal, vol. 45, pp. 487-488, 1953.

[13] P. Singh, "Broadening the genetic base of common beans cultivars: a review," Crop Science, vol. 41, pp. 1659-1675, 2001.

[14] W. Allard, "Sequential path analysis of grain yield and itscomponents in maize," Plant Breed, vol. 115, no. 5, pp. 343-346, 1960.

[15] A. Johnson and W. Wichern, Applied Multivariate Statistical Analysis, vol. 88, pp. 36-40, Prentice Hall, New York, 2nd edition, 1988.

[16] S. Falconer and C. Mackay, Introduction to Quantitative Genetics, Longman Scientific and Technical, New York, 4th edition, 1996.

[17] R. Weber and R. Moorthy, "Heritable and nonheritable relationship and variability of oil content and agronomic characters in the F2 generations of soybean crosses," Agronomy Journal, vol. 44, pp. 202-209, 1952.

[18] Statistical Analysis System, Institute SAS User Guides, Version 9.1, pp. 102-106, SAS Inc., Cary, NC, 2004.
[19] A. Shahid and C. Kamaluddin, "Correlation and path analysis for agro- morphological traits in Rajmash bean under BaramullaKashmir," African Journal of Agricultural Research, vol. 8, no. 18, pp. 2027-2032, 2013.

[20] A. Fahad, K. Muhammad, A. Obaid, A. Mukhtar, and C. Arshad, "Agro-morphological evaluation of some exotic common bean (Phaseolus vulgaris L.) genotypes under rain fed conditions of Islamabad, Pakistan," Pakistan Journal of Botany, vol. 46, no. 1, pp. 259-264, 2014.

[21] T. Sinclair, "Historical changes in harvest index and crop nitrogen accumulation," Crop Science, vol. 38, no. 3, pp. 638-643, 1998.

[22] S. Sivasubramanian and P. Madhavamenon, "Combining ability in rice," Madras Agricultural Journal, vol. 60, pp. 419-421, 1973.

[23] B. Alemayehu, "Genetic variation for grain yield and water absorption in common bean (Phaseolus vulgaris L.)," African Journal of Food Science Technology, vol. 1, no. 6, pp. 128-131, 2010.

[24] S. Ahmed and A. Kamaluddin, "Correlation and path analysis for agro-morphologicaltraits in rajmash beans under BaramullaKashmir region," African Journal of Agricultural Research, vol. 8, no. 18, pp. 2027-2032, 2013.

[25] W. Amir, B. Mohd, and M. Saba, "Morphological characterization as indices for yield and yield components allection in common bean (Phaseolus Vulgaris L.)," Journal Global Biosciences, vol. 4, no. 9, pp. 3391-3394, 2015.

[26] B. Berhanu, Genetic Variability and Character Associations In Bread Wheat (Triticum aestivum L.) Genotypes Developed For Semiarid Areas, Alemaya University of Agriculture, 2004.

[27] A. Kumar, S. Kumar, and K. Pal, "Genetic variability and character association for fruit yield and yield traits in cucumber," Indian Journal of Horticulture, vol. 65, no. 4, pp. 423-428, 2010.

[28] K. Baranwal, K. Mishra, K. Vishwakarma, S. Yadav, and B. Arun, "Studies on genetic variability, correlation and path analysis for yield and yield contributing traits in wheat," Plant Archives, vol. 12, no. 1, pp. 99-104, 2012.

[29] E. Ejigu, M. Wassu, and A. Berhanu, "Genetic variability, heritability and expected genetic advance of yield and yield related traits in common bean genotypes (Phaseolus vulgaris L.) at Abaya and Yabello Southern Ethiopia," African Journal of Biotechnology, vol. 17, no. 31, pp. 973-980, 2018.

[30] W. Johnson, F. Robinson, and E. Comstock, "Estimation of genetic and environmental variability in soybeans," Agronomy Journal, vol. 47, pp. 314-318, 1955.

[31] Z. Kifle, M. Firew, and D. Tadesse, "Estimation of association among growth and yield related traits in bread wheat (Triticum aestivum L.) genotypes at Gurage Zone Ethiopia," International Journal of Plant Breeding and Crop Science, vol. 3, no. 2, pp. 126-132, 2016.

[32] A. Kumar, K. Reddy, R. Pandravada, D. Rani, and V. Chaitanya, "Phenotypic variability, correlation and path coefficient analysis in pole type french beans (Phaseolus vulgaris L.)," Plant Archives, vol. 14, no. 1, pp. 313-319, 2016.

[33] S. Ebrahimnejad and V. Rameeh, "Correlation and factor analysis of grain yield and some important component characters in spring bread wheat genotypes," Cercetari Agronomice in Moldova, vol. 1, no. 165, pp. 5-15, 2016.

[34] A. Sadeghi, K. Cheghumirza, and R. Dorri, "The study of morphoagronomic trait relationship in common bean (Phaseolus vulgaris L.)," Biharean Biologist, vol. 5, no. 2, pp. 102-108, 2011.

[35] W. Emishaw, Comparison of the Growth, Photosynthesis and Transpiration of Improved and Local Varieties of Common bean (Phaseolus vulgaris L.) at Haramaya, Unpublished M. S. Thesis, College of Agriculture, School of Graduate Studies, Haramaya University, Haramaya, Ethiopia, 2007. 